\title{
Analysis of piano accompaniment to Sax
}

\author{
Wang Yue ${ }^{1, a}$ Xie $Y u^{1, a}$ \\ ${ }^{1}$ Qinhuangdao Institute of Technology, Qinhuangdao Hebei 066100, China \\ awyue_wang@163.com
}

Keywords: Sax; piano accompaniment; analysis

\section{Abstract:}

With the development of various industries in our country, musical instruments industry has a great development in recent years, various styles of musical instruments emerge in an endless stream. Piano music is very popular in Chinese, it is mainly pop music arranger, in the field of music is very important. The piano has "the king" reputation, not only its own expressive, but it is still a good accompaniment of musical instruments, especially for Sax, greatly improving the appeal of Sax. This paper mainly expounds the specific skills of the piano accompaniment for Sax.

Piano music has its own language style, through the piano it can express a kind of mysterious music color and more concise style of music. Sax and the piano has its own expressive force and the music expression effect. The frequency made by Sax is very close to the human voice frequency, therefore, it is welcomed and loved by many people. With the continuous development and innovation of music, many different kinds of music skills are innovated by people, in piano accompaniment to Sax, it has a high requirement for piano, and needs to be consistent with the connotation rhythm of music. The piano accompaniment to Sax can reflect the beauty of piano accompaniment and sound mixing.

\section{Accompaniment should have the characteristics of symphonic music}

In the accompaniment, there are a lot of complete piano accompaniment and concerto for Sax solo, such as "Matsulaki", "Christon" and "Roson" and " Ibele " etc. The piano accompaniment should exhibit the piano foil and assistance, and also should do the simulated performance of the symphony orchestra, to perform the sound of Orchestra. Making the piano accompaniment to have symphony effect, can promote the effect of the piano accompaniment to great extent, this requires a piano accompanist to imagine the used instrument timbre, to achieve the effect of symphonic music.

\section{The emotion performance of piano accompaniment and Sax sings}

Any music are through a certain form of texture to be performance. The so-called texture, refers to the structure style of longitudinal and transverse combination of different sound (lines) in the music works ${ }^{[1]}$. In the art songs, piano accompaniment and Sax's statement are more closed, it portrays Sax's artistic image, depicts the artistic conception of song, foils the mood of the Sax performer, and enriches the content of the work. The complex music texture fabricated by every kind of music morphology, volume, speed, timbre and tones etc. is the materialization of feelings, but also arouse the same move in our hearts ${ }^{[2-4]}$. Piano music is an excellent carrier acts as a representative symbol function, with its symbolic function accurately and properly to convey the mood of poetry, playing a leading and supplement role respectively on the theme, emotion and meaning of the work. 
When Sax learners in singing art songs, who should be clear the meaning of the performance of the piano accompaniment texture, their roles in the song's role and the traits of character of role ${ }^{[4-7]}$, yourself standing in what kind of environment atmosphere, in the quiet and sweet dreams, the twilight and soft moonlight, or in the quiet river to listening the bells ring. Moreover, with the change of accompaniment texture, the accompanist should accurately express the thoughts and

feelings of the songs ${ }^{[8,9]}$. In a sense, the piano accompaniment texture plays a decisive role in the Sax's art content and expression of emotion.

\section{Accompaniment needs to meet the connotation of the work}

In the accompaniment for Sax, the greatest degree of piano accompaniment on the need to comply with the connotation of the works. So, do a full interpretation of the music score of accompaniment music is the first step to meet the connotation. The language of music score is transformed into a heart of emotional expression, then into the inner sense of hearing, the hearing is unreal, just a kind of imagination. High quality accompaniment requires through the needs of sound and musical expression to make accompaniment. To grasp the timbre needed to express in music performance, complete the timbre of the grip through the touch-key. Piano vocal is related to the weight of hammer, different touch-key makes the different speed of hammer striking, and the timbre is not the same, by showing the rich and colorful sound reach the symphonic accompaniment sound, therefore, touch-key is very important. There are three main methods to touch the keys.

\section{A Legato key touching to express singing touch-key}

In the accompaniment of the singing melody, the melody is the core part of the charm music, if you want to take this good melody through playing the way to show, you need to use legato key touching method. For example, in the music "narrative poem", piano is mainly used in the theme melody of its' prelude, which can express a soft feel, to play this sweet feeling needs to use legato key touching way. Legato methods to touch the keys include: 1 . the general use of finger face to touch keys, mainly by reducing finger height to achieve a coherent effect; 2 . fingers to touch keys is coherent, but the action is slow and coherent; 3. the arm weight play, grasping the arm transverse moving forces, the vertical forces should be reduced, the purpose is to produce a certain buffer.

$B$ The touch-key way of interrupt play in leaps and staccato

In the accompaniment, the staccato or leaps are applied to achieve the plucked effect of string music. The notes of both staccato and leaps have very big distinction, tone leaps is half of full notes, staccato is slightly shorter than leaps. Touch-key of interrupt play has fast playing speed, staccato's touch-key is faster than the speed of leaps in general. To improve the imitated pizzicato effect, the accompanist will use the way of interrupt play touch-key to play staccato and leaps. the touch-key mode of staccato has a great demand for speed, the time of finger touching the keys should be relatively short, the speed of keys' up and down should be faster, and moreover should reduce the touch area of touch-key, which requires the very sensitive fingers.

\section{Volume control of accompaniment}

\section{A Volume of each register in the accompaniment}

Because different timbres in Sax's register is different, in the piano accompaniment, it should be careful to note Sax tone's change in different ranges, through the timbre change to control the tempo piano, for example, the timbre of human's is very similar to the Sax's alto district, and euphemism, in this, it needs to control a good volume in accompaniment, cannot higher than the 
sound of Sax, and it should highlight the sound of Sax, through the piano accompaniment, foil the charm tone of Sax in Sax playing to the maximum extent.

$B$ Opening volume in interlude and legato

In the interlude and legato, it can be according to the work style and different requirements, increasing the accompaniment volume, the size of the volume needs to be just perfect. Legato has a kind of effect of come to the point, can attract the audience to a certain extent. Interlude is mainly to make the connection between the passages, making the player smoothly immersed in music emotion.

C Accompaniment should be in a subordinate position

Accompaniment volume should be less than the theme melody volume, to highlight the solo melody, so as to reach a perfect effect. For example, in the "Ibele", the theme melody is the Sax, the music is exquisite and pleasant to listen to, in piano accompaniment, it has very high request to two hands plucked of the accompanist. the touching keys sound of left and the right hand imitate violin pizzicato and Cello bass respectively, both hands weight should be as low as possible, mainly highlights Sax's main melody.

D Implementation of volume control on the basis of the different music

In accompaniment, the size of the volume is according to the different forms of music to grasp reasonably, such as, in the end sounds, it is usually through the treble area of Sax to show surging, at this time, the accompaniment needs to amplify in to a certain extent, mainly to a foil for Solo, enhancing the sound of more gorgeous part of solo.

\section{The accurate intensity level design}

The greatest charm of piano is intensity level design, which can highlight the strength of music performance. The music had strong expressive force required by the accurate strength and intensity level design to reasonably express the emotion. The emotion of creators will not through single sound intensity to express, this strength of single expression form cannot reach the expected effect of infection. The same melody played by different intensity, can enhance the appeal of music to a great extent. Therefore, the reasonable planning of intensity level is very important, generally includes the following aspects.

\section{A Intensity level design in each voice part}

If the intensity of the accompaniment and melody is very close, so that there is no clear stratification between the two layers, this music is just like the picture which does not have the level of the sense, in general making people feel uncomfortable, so, it should have different intensity levels between each layer, as a key design in the design, it is very much important to the overall sense of the whole music.

(a) the intensity level arrangement of main melody and accompaniment are as follows: 1 The main melody mainly contains the first melody in high vocal register and repeat eight degree voices, these voices were stronger in performance as strength as possible, play must be relaxed, arm weight is shifting to the playing hands and fingers. At the same time, palms, fingers and wrists should support. 2. The playing strength of accompaniment part should be according to the change of specific circumstances to be lighter, and should reduce intensity of touch keys.

(b) In playing polyphony music, it needs to hold the balance between the various parts, for example, in "Guerra R Andrianof", it needs for attention to counterpoint music. in theme playing, intensity level design must have outstanding, in counterpoint, by touching the keys in many different ways and adjust the intensity design of touch-key to do the counterpoint playing, and 
targeting to distinguish the voice color and voice lines.

$B$ The intensity level design

Each time point of the different music expression and appeal results in different music performance strength, the expressive force of music through music back push to reasonably arrange for intensity. This intensity arrangement is changing, in many cases, the strength of music's beginning and the end is weak, but the music's high spot is strong. When the melody is back pushed, the strength changes constantly and gradually enhances. With the constantly advancing of the climax of the music, strength increased substantially, the music tends to flat and in after the climax. These changing intensity need according to the style of the creators, whatever the changes, in intensity design, it should be based on the requirements of music works.

In music, the sound has strong and weak points, but the intensity of emotional expression which do not exist in isolation, and music are closely linked, intensity change arrangements are basis can be found, he random changes makes music to lose infectivity, intensity changes cannot be accomplished by no credentials of the imagination, the intensity level design shall be designed according to the technique of expression and emotion in mood and other requirements.

\section{Problems of piano accompaniment should pay attention to}

First of all, the pianist must make analysis of the full band score firstly, and understand which is the corresponding musical instrument imitated by every melody line in piano accompaniment, so as to accomplish a definite object in view. Understanding the band score to imitate every part of the instrument effect, so how to imitate and is a very important problem, which requires players to master the skills of controlling tone. It mainly includes two aspects of key touching techniques and use of pedal skills, these two aspects are the most direct means to control the timbre.

A Effect of touch keys on timbre.

In the accompaniment, the way of touch-key, can directly change the piano sound. The common way has high lift finger and fast touch-key, low lift finger and fast touch-key, fingertip touch-key, elbow force touch-key and seamless coherence touch-key, etc. The above are some of the common ways of touch-key, through the change of strength, speed, angle, radian, height, depth of touch-key, they give out different tones, to better mimic different sound effect of bands' instrument.

$B$ The effect of use of pedal on timbre

On the piano performers, pedal likes a palette of painter's hands, with the different methods to touch the keys, will play a different sound effects. the ways of using pedal includes: the long pedal, so that the sound is vigorous; the shallow pedal, the step depth is only $1 / 2,2 / 3,3 / 4$ and so on; and fibrillation pedal, refers to the step way of ankle jittering or when plays the fast phrase, each syllable should change the pedal one time, when plays soft pianissimo, step on the soft pedal. The use of pedal is very delicate, with a pedal, different intensity of trampling has different sound effects. Application of the myriads of changes of the pedal, which requires the performers with hearing to distinguish. According to the different timbre needs, different ways of stepping are chosen.

\section{The necessary comprehensive quality of accompanist}

Good technical literacy, theoretical literacy, cultural literacy, psychological quality and occupation literacy is a necessary condition for piano accompanist to play good accompaniment, and realize the perfect combination of mind and skill. The author is engaged in the piano accompaniment for many years, and has accumulated a lot of experiences in this aspects, here is 
some little humble opinions, and the author would like to share them with the peers and experts in the piano accompaniment field.

\section{A Technology literacy}

Compared with the solo, piano accompaniment is worth us studying carefully for its unique technical problems. The author thinks that the diversification of the fingers touch-keys, the reasonable degree of the use of pedal, the effective regulation and control of speed, rhythm and accompaniment volume and the ability to quickly grasp the music, are mainly reflected the technology literacy of a piano accompanist. Below, the example, the piano accompaniment of the first movement of "Violin Sonata in A major" is used to illustrate the close relationship between the art expressive force and method to touch-key.
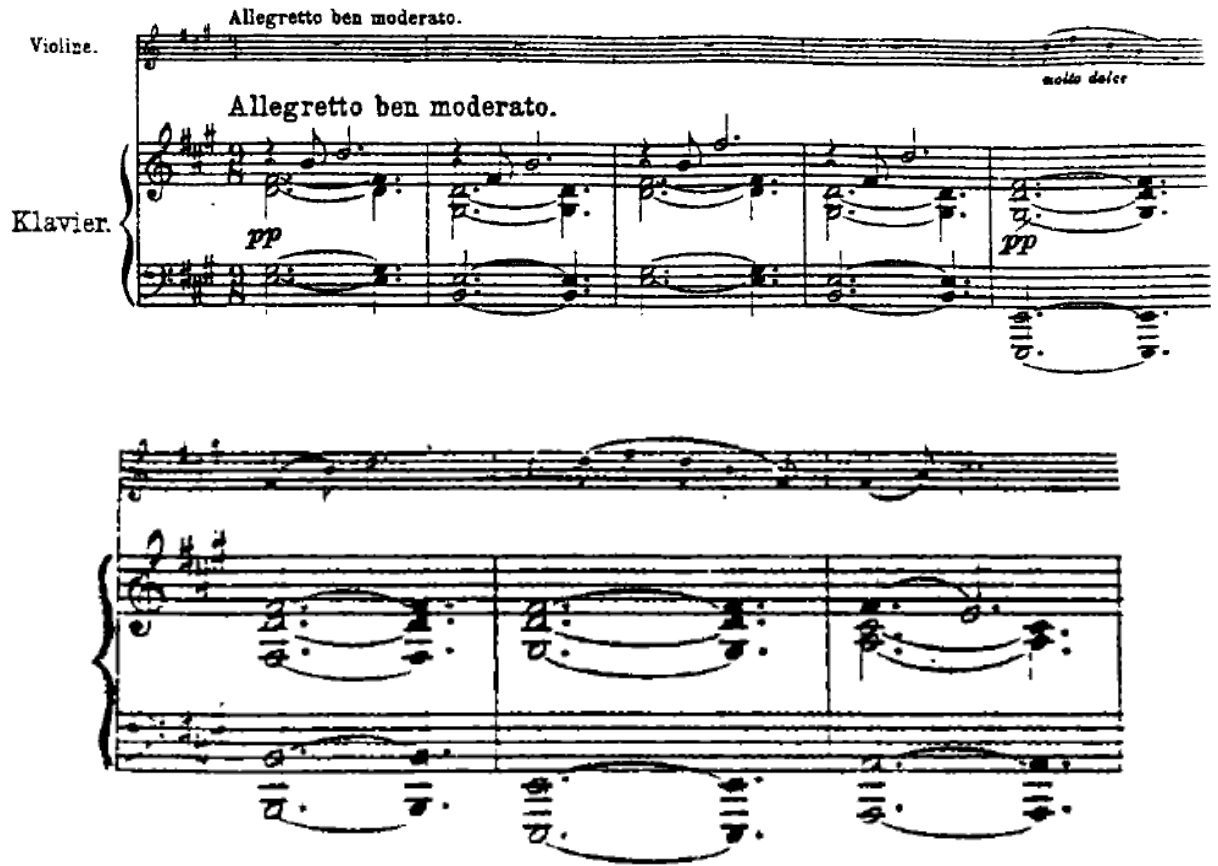

Figure 1 first movement of "Violin Sonata in A major"

The lyrical melody of the movement is charming. The music started, the prelude of 4 sections are played by the piano, expressing a very quiet atmosphere, and then leading to the affectionate pour out of violin. To accurately represent this artistic conception, it requires the piano accompaniment can also like a violin as a singing voice. To obtain this sound, it must be careful about where and how the fingers touch-key, it needs soft finger to slowly touch-key, while the use of weight of the arm and the rotation of wrist, finger is as close as possible to the key surface as smooth move, to avoid knocking, and reducing the fingers' motion transformation traces to a minimum. From the above, we can clearly recognize that: and the position of key touching and how many parts of key touching surface of fingers in the accompaniment, the speed, intensity and depth of using finger makes keys to be down, will have an effect on the light and shade, strength, thickness and even far and near of sound in varying degrees. While the technology literacy of piano accompanist is also reflected in the artistic skill of fingers.

\section{$B$ The theoretical attainment}

The theoretical attainment of music is an important condition to guide the implementation process of the accompaniment. The so-called theoretical attainment, refers to the rational knowledge of accompanist's interpretation and reproduction to the structure of music and music works, it is a dominant music aesthetic mechanism. In view of the professional piano 
accompaniment involved many theory knowledge of music, so how about the accompanist music theory accomplishment, will be directly related to the quality and taste of the accompaniment.

\section{Conclusion}

When using the piano accompaniment for Sax, accompanist must always pay attention to the breathing of Sax bassoonist. The breathing of Sax bassoonist depends on the phrase length, so the integrity of the phrase should to be maintained in the accompaniment. Accompanist should be according to the breath length of oboist, the need of phrase to determine the opening position. No matter how changes in the rhythm of the music and the length of breath, accompanist and performer must according to the need of the connotation expression of the works and make the common breath, which can cooperate tacit. The two instruments of piano and Sax cooperate each other to make music innovation, can enhance the appeal of Sax solo largely.

\section{References}

[1] Wu Longyan. Analysis of piano accompaniment skills for Sax [1] [J]. Northern music, 2012, 03:16-18.

[2] Li Miao. Origin and schools of classical saxophone [J]. Journal of Shenyang Normal University (SOCIAL SCIENCE EDITION), 2013, 01:180-182.

[3] Xu Zhu, GLEE. Modern Fusion guitar full contact (nine) -- to expand ideas (2nd): vocabulary of learning other instruments, [J]. Instruments, 2009, 05:88-91.

[4] Jiang Xiangdong. Analysis of Ravel art songs with piano accompaniment [J]. Symphony. Journal of Xi'an Conservatory of Music, 2006, 02:67-70.

[5] Liu Peng. Analysis of Pipa Concerto "blessing" with Piano accompaniment [J]. Qilu art, 2013, 05:35-38.

[6] Xu Xiaowei. Analysis of the main aria of Chinese contemporary opera "struggle in an ancient city" with piano accompaniment [J]. Art education, 2012, 04: 94.

[7] Gong Aosiyao. Research on the artistic characteristics and piano accompaniment of Schuhmann's vocal divertimento "Dichterliebe" [D]. Hunan Normal University, 2012

[8] Qu Feng. Translation and review of "the piano music in nineteenth Century" [D]. Shanghai Conservatory of Music, 2012

[9] Guo Jie. On the expressive function of piano accompaniment for vocal music [J]. Journal of Xinghai Conservatory of Music, 2006(4): 90-92 